\title{
Multifed Printed Reflectarray With Three Simultaneous Shaped Beams for LMDS Central Station Antenna
}

\author{
Manuel Arrebola, Member, IEEE, José A. Encinar, Member, IEEE, and Mariano Barba
}

\begin{abstract}
A two-layer reflectarray is proposed as a central station antenna for a local multipoint distribution system (LMDS) in the 24.5-26.5 GHz band. The antenna produces three independent beams in an alternate linear polarization that are shaped both in azimuth (sectored) and in elevation (squared cosecant). The design process is divided into several stages. First, the positions of the three feeds are established as well as the antenna geometry to produce the three beams in the required directions. Second, the phase distribution on the reflectarray surface, which produces the required beam shaping, is synthesized. Third, the sizes of the printed stacked patches are adjusted so that the phase-shift introduced by them matches the synthesized phase distribution. Finally, the radiation patterns are computed for the central and lateral beams, showing a shaping close to the requirements. A breadboard has been manufactured and measured in an anechoic chamber, showing a good behavior, which validates the designing methodology.
\end{abstract}

Index Terms-Local multipoint distribution system (LMDS), multibeam antenna, multifeed reflectarray, reflectarray, shaped beam antenna.

\section{INTRODUCTION}

$\mathbf{P}$ rinted reflectarrays are very attractive solutions since they have a number of advantages such as, low profile, mass and volume, they are easy to manufacture and offer possibilities for beam shaping and electronic beam control, [1] and [2]. A reflectarray consists of a planar array of printed elements illuminated by a primary feed, typically a horn antenna. Each element of the reflectarray introduces a phase-shift to the impinging wave from the feed to produce a focused or contoured beam. The applications for contoured beam reflectarrays are especially interesting since the manufacturing cost and process is the same as for a focused beam. Shaped beam reflectarrays were demonstrated using patches with attached stubs [3] and varying-sized patches [4] as phasing elements. In both cases, the narrow band of the reflectarray elements based on a single layer of patches, limits the antenna performance. This limitation can be overcome by using stacked patches of varying sizes [5], [6]. Shaped beam re-

Manuscript received November 8, 2007; revised February 7, 2008. This work was supported in part by the Spanish Ministry of Education and Science under project CICYT TEC2004-02155.

M. Arrebola is with the Electrical Engineering Department, Universidad de Oviedo, E-33203, Gijón (Asturias), Spain (e-mail: arrebola@tsc.uniovi.es).

J. A. Encinar and M. Barba are with the Electromagnetism and Circuit Theory Department, Universidad Politécnica de Madrid, E-28040 Madrid, Spain (e-mail: encinar@etc.upm.es).

Color versions of one or more of the figures in this paper are available online at http://ieeexplore.ieee.org.

Digital Object Identifier 10.1109/TAP.2008.923360 flectarrays have been also demonstrated in folded configurations using one and three layers of varying-sized patches [7], [8].

Multiple-beam antennas are used in radar and communications applications, being typically based on large phased arrays [9]-[11] or reflectors with feed-horn clusters and mechanical devices [12]. Reflectarrays can be a suitable technology for multibeam antennas, because of their low-cost and low-complexity advantages, particularly when shaped beams are required. Several works on multiple-beam reflectarrays have been published up to now considering one feed per beam, but most of them are for focused beams [13]-[15]. Two concepts have been demonstrated to generate simultaneous shaped beams, but restricted to single linear polarization; the first one is based on an independent beam for each polarization [16], while the second one requires a subreflectarray in a folded configuration [13]. A single offset reflectarray has been demonstrated for real DBS (Direct Broadcast Satellite) mission requirements [16]. In this case, the reflectarray was designed to produce two beams in orthogonal polarizations, a shaped beam in horizontal polarization and a pencil beam in vertical polarization, by an independent adjustment of the phase for each polarization. On the other hand, the multibeam folded reflectarray in [13] was designed using the principle of a bifocal antenna, where a focal ring replaces the focal point, by using an additional phasing on the polarizing radome. More recently, the bifocal concept was combined with a phase-only synthesis to design a folded reflectarray with three sector beams [17]. In this antenna, the three beams are independent, shaped in one dimension and work in the same linear polarization. Folded reflectarrays are very compact antennas but they can only work in a single linear polarization because of the inclusion of a polarizer as a subreflector.

Up to the author's knowledge, reflectarrays with simultaneous shaped beams have only been demonstrated for the above mentioned configurations, which imply a polarization constraint. Using the technique described in [16] only two beams can be generated, one in each orthogonal polarization; on the other hand, several beams can be produced using a folded reflectarray [17], but only in the linear polarization defined by the polarizing grid subreflector.

In this paper, a passive reflectarray with three simultaneous shaped beams is demonstrated considering several alternatives for the polarization of each beam. A reflectarray made of two layers of varying-sized patches has been designed for a central station of Local Multipoint Distribution Service (LMDS) in the 24.5-26.5 GHz band, considering the two linear polarizations. The antenna required for this application should have a 


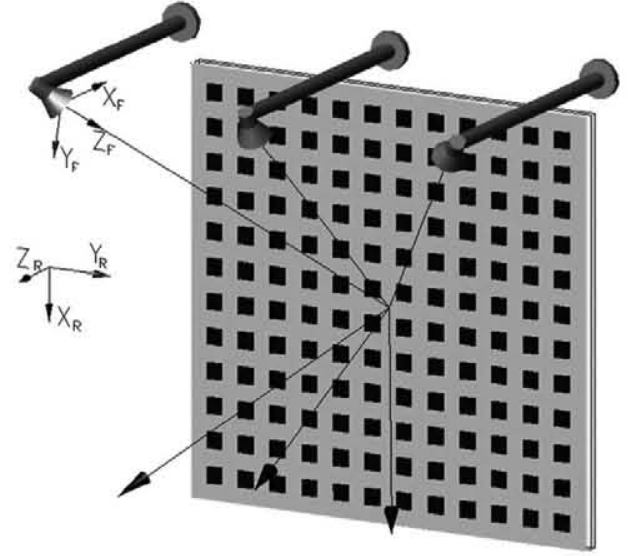

Fig. 1. Scheme of the proposed multifeed reflectarray.

shaped beam, sectored in azimuth and squared cosecant in elevation [18]. A reflectarray with three $30^{\circ}$-sector beams in azimuth is proposed as an LMDS central station antenna with an overall coverage of $90^{\circ}$, which allows an increase of the system capacity with respect to a single $90^{\circ}$-sector antenna. The reflectarray has three feeds in fixed positions and each one is related to only one sector beam. The design process is carried out for dual linear polarization, vertical (V) and horizontal (H), so that the beams can be generated in any polarization. The application requires only linear polarization, so an alternate polarization plan is proposed for the three azimuth sectors, in order to reduce the co-channel interference between adjacent sectors. Therefore two options are considered for the polarization of the three beams, V-H-V and H-V-H. The antenna has been manufactured and tested for both polarization options, and the results show a good agreement with the shaping requirements.

\section{ANALYSIS AND DESIGNING PRINCIPLES}

The scheme of a multifeed printed reflectarray in the proposed configuration is shown in Fig. 1. In principle, horn antennas are considered as primary feeds. In general, the reflectarray is a multilayer structure of printed stacked patches over a ground plane. Since the antenna has three independent beams, each one related to only one feed and the reflectarray is analyzed separately for each feed. For the analysis, two coordinate reference systems are considered: the feed reference system, $\left(\boldsymbol{x}_{f}, \boldsymbol{y}_{f}, z_{f}\right)$, which is different for each feed and centered at its phase-centre; and the reflectarray reference system, defined by $\left(\boldsymbol{x}_{r}, \boldsymbol{y}_{r}, \boldsymbol{z}_{r}\right)$ and whose origin is in the centre of the reflectarray surface, see Fig. 1.

In the following, the expressions are given for one of the feeds, but they are exactly the same for the other feeds. First, the incident field $\boldsymbol{E}_{\text {inc }}^{X / Y}$ on each reflectarray cell produced when one feed illuminates the reflectarray is computed. Since two linear polarizations, $X_{\mathrm{R}}$ and $Y_{\mathrm{R}}$, can be considered, the super index $X / Y$ denotes the polarization defined by the feed. For each polarization, the two components of the field tangential to the reflectarray surface are calculated, so that the incident field is

$$
\boldsymbol{E}_{\mathrm{inc}}^{X / Y}=E_{x \text { inc }}^{X / Y} \boldsymbol{x}_{r}+E_{y \text { inc }}^{X / Y} \boldsymbol{y}_{r} .
$$

Note that for $X_{\mathrm{R}}(V)\left[Y_{\mathrm{R}}(H)\right]$, an ideal linear polarized feed will produce mainly a field in the $X_{\mathrm{R}}$-direction [ $Y_{\mathrm{R}}$-direction], but also a small component in the $Y_{\mathrm{R}}$-direction $\left[X_{\mathrm{R}}\right.$-direction] will be produced out of the principal planes. In a similar way, the field reflected on the reflectarray surface can be written as

$$
\boldsymbol{E}_{\text {ref }}^{X / Y}=E_{x \text { ref }}^{X / Y} \boldsymbol{x}_{r}+E_{y \text { ref }}^{X / Y} \boldsymbol{y}_{r} .
$$

For the calculation of the reflected field, the analysis of the reflectarray is carried out element by element considering local periodicity. The cross polarization introduced by the patches is considered, so that the reflected field for each element $(m, n)$ is

$$
\boldsymbol{E}_{\mathrm{ref}}^{X / Y}(m, n)=\boldsymbol{R}(m, n) \cdot \boldsymbol{E}_{\mathrm{inc}}^{X / Y}(m, n)
$$

where

$$
\boldsymbol{R}=\left(\begin{array}{cc}
\rho_{x x} & \rho_{x y} \\
\rho_{y x} & \rho_{y y}
\end{array}\right) .
$$

The components of the matrix are complex and characterize the behavior of the reflectarray element. The components $\rho_{x x}$ and $\rho_{y x}$ are the direct and cross reflection coefficients for an incident wave with the electric field in the $X_{\mathrm{R}}$ direction. On the other hand, $\rho_{y y}$ and $\rho_{x y}$ are the reflection coefficients for the $y_{r}$ component. All of them depend on the angle of incidence of the impinging wave coming from the feed, and on the element geometry, adjusted to produce the required phase-shift, so that the matrix is different for each element of the reflectarray, and also for each feed in the case of a multifeed reflectarray (because the angle of incidence changes). As a result, each reflectarray element has to be analyzed independently using a full-wave method. Here, the reflectarray analysis is based on the method of moments (MoM) in the spectral domain assuming local periodicity. Under this approach, each element is analyzed considering the real dimensions and incident field (polarization and angle of incidence) but assuming a periodic environment. This approach is accurate when the variation of the dimensions from element to element is smooth, and it has been validated by experimental results for a contoured-beam reflectarray [16]. The analysis technique, described in [19], computes the total field reflected by the reflectarray elements as the superposition of two components: the field reradiated by the patches, and the field reflected by the grounded dielectric structure (when the patches are not present).

Once the total field reflected by the reflectarray elements has been calculated, the radiation pattern of the antenna is computed. Assuming the second principle of equivalence, the components $\theta$ and $\varphi$ of the radiated far field are given by

$$
\begin{aligned}
& E_{\theta}^{X / Y}(u, v)=j k_{0} \exp \left(-j k_{0} r\right)\left(P_{x}^{X / Y}(u, v) \cos \varphi+P_{y}^{X / Y}(u, v) \sin \varphi\right) \\
& 2 \pi r
\end{aligned}
$$

$$
E_{\varphi}^{X / Y}(u, v)=-j k_{0} \exp \left(-j k_{0} r\right) \cos \theta
$$

$$
\frac{\left(P_{x}^{X / Y}(u, v) \sin \varphi-P_{y}^{X / Y}(u, v) \cos \varphi\right)}{2 \pi r}
$$


where $u$ and $v$ coordinates are defined as

$$
u=\sin \theta \cos \varphi ; \quad v=\sin \theta \sin \varphi
$$

and $P_{x}^{\mathrm{X} / Y}(u, v)$ and $P_{y}^{X / Y}(u, v)$ are the radiation integrals throughout the reflectarray aperture $(R A)$

$$
\begin{gathered}
P_{x}^{X / Y}(u, v) \\
=\iint_{R A} E_{x \text { ref }}^{X / Y}\left(x_{r}, y_{r}\right) \exp \left[j k_{0}\left(u x_{r}+v y_{r}\right)\right] \\
\cdot d x_{r} d y_{r} \\
P_{y}^{X / Y}(u, v) \\
=\iint_{R A} E_{y \text { ref }}^{X / Y}\left(x_{r}, y_{r}\right) \exp \left[j k_{0}\left(u x_{r}+v y_{r}\right)\right] \\
\cdot d x_{r} d y_{r} .
\end{gathered}
$$

Finally, starting from the components calculated using (5) and (6), the co and cross polar radiations patterns given by Ludwig's third definition can be obtained [20].

The reflected field is calculated at the centre of each reflectarray element placed on a regular mesh of period $\Delta x$ and $\Delta y$ in the $X_{\mathrm{R}}$ and $Y_{\mathrm{R}}$ directions. If the field is assumed to be constant on the surface of each reflectarray cell $(m, n)$, the double integral of (8) and (9) can be written as a double sum as follows:

$$
\begin{aligned}
P_{x}^{X / Y}(u, v)= & K \cdot \sum_{m} \sum_{n} E_{x \mathrm{ref}}^{X / Y}(m, n) \\
& \cdot \exp \left[j k_{0}(u m \Delta x+v n \Delta y)\right] \\
P_{y}^{X / Y}(u, v)= & K \cdot \sum_{m} \sum_{n} E_{y \mathrm{ref}}^{X / Y}(m, n) \\
& \cdot \exp \left[j k_{0}(u m \Delta x+v n \Delta y)\right]
\end{aligned}
$$

where $K$ is a constant

$$
K=\Delta x \Delta y \sin c\left(\frac{k_{0} u \Delta x}{2}\right) \operatorname{sinc}\left(\frac{k_{0} v \Delta y}{2}\right) .
$$

In (10) and (11) the double sum can be written as a Two-dimensional Inverse Discrete Fourier Transform (IDFT2) so that $P_{x}^{X / Y}$ and $P_{y}^{X / Y}$ are calculated as

$$
\begin{aligned}
& P_{x}^{\mathrm{X} / Y}(p, q)=N_{x} N_{y} K \cdot I D F T 2\left(E_{x \text { ref }}^{\mathrm{X} / Y}(m, n)\right) \\
& P_{y}^{\mathrm{X} / Y}(p, q)=N_{x} N_{y} K \cdot I D F T 2\left(E_{y \text { ref }}^{X / Y}(m, n)\right)
\end{aligned}
$$

where indexes $p$ and $q$ are related to $u$ and $v$ as

$$
u=\frac{2 \pi p}{N_{x} \Delta x k_{0}} ; \quad v=\frac{2 \pi q}{N_{y} \Delta y k_{0}} .
$$

If (3) is developed for $X_{\mathrm{R}}$ linear polarization, the $x_{r}$ and $y_{r}$ components of the reflected field for an impinging wave at each reflectarray element are given by

$$
\begin{aligned}
& E_{x \mathrm{ref}}^{X}=\rho_{x x} \cdot E_{x \mathrm{inc}}^{X}+\rho_{x y} \cdot E_{y \text { inc }}^{X} \\
& E_{y \mathrm{ref}}^{X}=\rho_{y x} \cdot E_{x \text { inc }}^{X}+\rho_{y y} \cdot E_{y \text { inc }}^{X} .
\end{aligned}
$$

In this case, (16) and (17) will produce the co and cross polar components respectively in Cartesian coordinates. In (16) the amplitude of the first term of the sum is much bigger than the second one

$$
\left|\rho_{x x} \cdot E_{x \text { inc }}^{X}\right| \gg\left|\rho_{x y} \cdot E_{y \text { inc }}^{X}\right|
$$

because the second term is the product of the cross-polarization terms introduced by the feed and by the reflectarray elements, so that the copolar reflected field can be written as

$$
E_{x \mathrm{ref}}^{X} \approx \rho_{x x} \cdot E_{x \text { inc }}^{X} .
$$

If (19) is introduced into (13), it remains as follows:

$$
P_{x}^{X}(p, q) \approx N_{x} N_{y} K \cdot \operatorname{IDFT2}\left(\rho_{x x}(m, n) \cdot E_{x_{\mathrm{inc}}}^{X}(m, n)\right) .
$$

In this equation, the $P_{x}^{X}$ function depends on an IDFT2 of a product, which can be written as the convolution of two IDFT2 as

$$
\begin{aligned}
P_{x}^{X}(p, q) \approx N_{x} N_{y} K\left[\operatorname{IDFT2}\left(\rho_{x x}(m, n)\right)\right. \\
\left.* I D F T 2\left(E_{x \mathrm{inc}}^{X}(m, n)\right)\right] .
\end{aligned}
$$

So, the copolar component of the radiation pattern can be viewed as the convolution of the IDFT2 of the reflection coefficient, $\rho_{x x}$, and the IDFT2 of the copolar incident field, $E_{x \text { inc }}^{X}$, on the reflector surface. The reflection coefficient of a reflectarray element depends on the angle of incidence of the impinging wave, but it does not vary much for incidence angles of less than 40 degrees. If the incidence angles remain below this value for all the feeds, the IDFT2 $\left(\rho_{x x}(m, n)\right)$ and the $\operatorname{IDFT2}\left(E_{x \text { inc }}^{X}(m, n)\right)$ functions can give the beam shaping and beam direction, respectively.

This point of view permits a shaped beam to be synthesized and the corresponding multifed reflectarray designed to obtain multiple shaped beams. The beams are obtained by moving and pointing the feed-horns so that they are independent and each beam is related to only one feed-horn.

On the other hand, (17) gives the crosspolar component of the reflected field. Here, the two terms of the sum correspond to two different contributions to the crosspolar radiation. The first term, $\rho_{y x} \cdot E_{x \text { inc }}^{X}$, is the contribution generated by the printed elements, while the second term, $\rho_{y y} \cdot E_{y \text { inc }}^{X}$, is the crosspolar caused by the geometry. In this case, the two terms of the sum have a similar magnitude and none of them is negligible.

Finally, an analogous discussion can be made for $Y_{\mathrm{R}}$ polarization by developing (3) for this polarization.

\section{ANTENNA DESIGN}

In order to validate the methodology described above, a multiple feed and multiple shaped-beam reflectarray has been designed, manufactured and measured. The reflectarray is designed to fulfill the coverage and gain requirements of an LMDS central station antenna, [18]. In this work, 90-degree 
sector coverage with three 30 -degree independent beams is proposed. Each beam is generated by a different horn antenna but the reflectarray is the same for all of them. A general scheme of the proposed antenna with three feeds and three shaped beams is shown in Fig. 1. Although only one linear polarization is required in an LMDS service, the reflectarray is designed to work in dual polarization and a configuration with beams in alternate polarization for adjacent beams is proposed, i.e., the central beam works in one linear polarization vertical $(\mathrm{V})$ or horizontal (H) while the lateral beams work in the orthogonal polarization. Thus, two configurations will be possible with the same reflectarray, $\mathrm{V}-\mathrm{H}-\mathrm{V}$ and $\mathrm{H}-\mathrm{V}-\mathrm{H}$. This configuration permits the capacity of the system to be increased, since polarization isolation between adjacent sectors is introduced. Note that $\mathrm{V}$ corresponds to $X_{\mathrm{R}}$-polarization, because the electric field in the $X_{\mathrm{R}}$-direction is vertical, according to the reference system shown in Fig. 1, and $\mathrm{H}$ corresponds to the $Y_{\mathrm{R}}$-polarization.

According to the designing principle stated at the end of Section II, the reflectarray is designed for one beam (central), so that the distribution of $\rho_{x x}(m, n)$ in (20) is implemented by adjusting the dimensions of the patches to produce the beam shaping, and the other beams (lateral) are obtained through a suitable placing of the feed horns. Since in this configuration the entire antenna is symmetric with respect to the $X_{\mathrm{R}} Z_{\mathrm{R}}$ plane, only one lateral beam will be evaluated.

\section{A. Antenna Definition}

A central station antenna for an LMDS application requires a shaped beam, squared cosecant in elevation and sectored in azimuth, see minimum and maximum requirements in Fig. 2 for 30-degree sectors with 5-degree tilt. The elevation masks are the same for all the beams, whereas the azimuth coverage depends on the sector. The three-dimensional masks are obtained through a combination of the elevation and azimuth requirements for each beam.

To fulfill the previous requirements in beam shaping and gain a $175 \mathrm{~mm} \times 175 \mathrm{~mm}$ reflector surface is defined. Since the bandwidth of the application is about $8 \%, 24.5$ to $26.5 \mathrm{GHz}$, a two-layer printed reflectarray with rectangular patches of variable size is considered [5]. The periodic cell is defined as 5.84 $\mathrm{mm} \times 5.84 \mathrm{~mm}$, which is half a wavelength at the central frequency, $25.5 \mathrm{GHz}$, so that grating lobes are avoided or negligible in the entire frequency band. As a result, the reflectarray is a squared flat panel with 30 elements $\times 30$ elements.

A standard horn antenna, FMI 20240 from Flann Microwave Inc., has been selected for the feeds and its pattern has been modeled as a $\cos ^{\mathrm{q}}(\theta)$ function. Since the feed is a pyramidal horn, the pattern in E-plane and H-plane is not the same in width so that different $q$ values are estimated in each plane and an averaged value is used in the model. The $q$-factor also depends on the frequency and has been calculated at the central and extreme frequencies see Table I.

The feed positions are selected by taking three conditions into account, according to the designing principle discussed in Section II. First, the illumination levels should be similar for the three feeds in order to obtain a similar gain for all of the beams. Also, if the illumination is very different for the central and the lateral feeds, the beam shaping will be distorted. Second, each

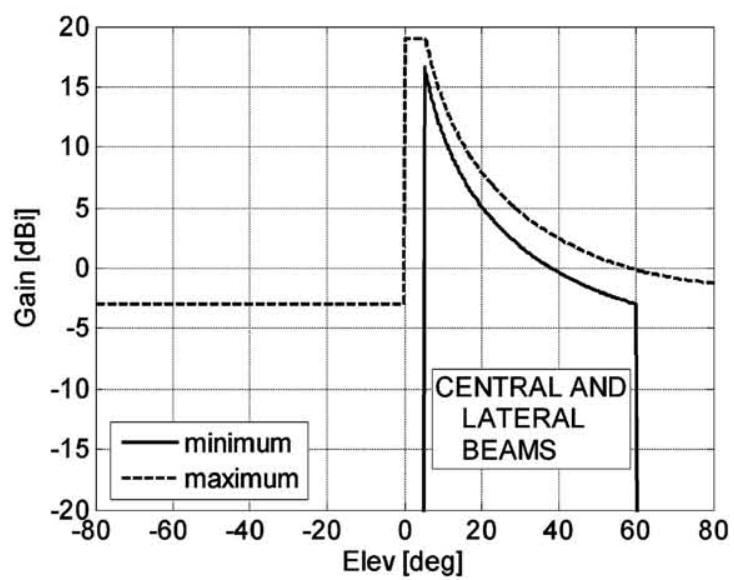

(a)

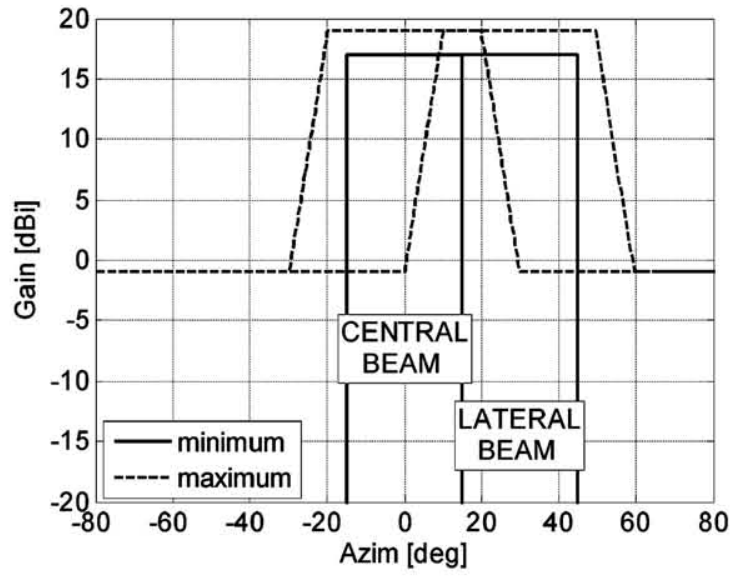

(b)

Fig. 2. Pattern requirements in elevation (a) and azimuth (b). Maximum (dashed lines) and minimum (solid lines) masks.

TABLE I

VALUES OF $\boldsymbol{q}$ FACTOR USED TO MODEL THE FEED HORNS

\begin{tabular}{cccc}
\hline Frequency (GHz) & 24.5 & 25.5 & 26.5 \\
\hline q-factor in E-plane & 33 & 35 & 37 \\
\hline q-factor in H-plane & 37 & 39 & 41 \\
\hline $\begin{array}{c}\boldsymbol{q} \text { in } \cos ^{\boldsymbol{q}}(\boldsymbol{\theta}) \\
(\text { averaged) }\end{array}$ & 35 & 37 & 39 \\
\hline
\end{tabular}

feed must generate a beam in the correct direction assuming an ideal metallic plane, so that the beam direction is mainly determined by the feed position, and the beam shaping by the phasing introduced by the stacked printed patches. Third, the incidence angles should not be very large, under 45 degrees, which guarantees that the reflection coefficient matrix of most reflectarray elements will be very similar for all the feed horns.

The positions of the three feeds and the pointing of the reflected beam are summarized in Table II. All the feeds point toward the centre of the reflectarray. The illumination produced by the central and lateral feeds has been computed and compared, showing quite similar levels. However, they cannot be exactly the same and this will cause a small distortion in the beam shaping. 
TABLE II

FEED HORN DATA

\begin{tabular}{|c|c|c|c|}
\hline & & Central feed & Lateral feeds \\
\hline \multicolumn{2}{|c|}{$\begin{array}{c}\text { Coordinates of the } \\
\text { phase-centre }\end{array}$} & $(-94,0,214) \mathrm{mm}$. & $(-94, \pm 113,182) \mathrm{mm}$ \\
\hline \multirow{2}{*}{$\begin{array}{l}\text { Pointing of } \\
\text { the reflected } \\
\quad \text { beam }\end{array}$} & $(E l, A z)$ & $(-23,0)$ deg. & $(-23, \pm 30)$ deg. \\
\hline & $(\theta, \varphi)$ & $(23,0)$ deg. & $(37, \pm 51)$ deg. \\
\hline \multicolumn{2}{|c|}{$f / D$} & 1.2 & 1.0 \\
\hline
\end{tabular}

Since the reflectarray is designed considering dual linear polarization, each feed has to be positioned so that the cross-polar field generated is reduced as much as possible. This point implies a rotation around the $Z_{\mathrm{F}}$ axis of the feed horn for the lateral beams, so that the polarization of the feed is either vertical or horizontal in the reflectarray reference system, fulfilling (22) or (23), respectively.

$$
\begin{aligned}
& \boldsymbol{y}_{r} \cdot \boldsymbol{E}_{f}=0 \\
& \boldsymbol{x}_{r} \cdot \boldsymbol{E}_{f}=0
\end{aligned}
$$

where $\boldsymbol{E}_{f}$ is the E-plane (H-plane) vector of the horn antenna, typically the $\boldsymbol{y}_{f}\left(\boldsymbol{x}_{f}\right)$ vector. When the beam directions are very close to the plane $X_{\mathrm{R}} Z_{\mathrm{R}}(\varphi \approx 0 \mathrm{deg})$ or $Y_{\mathrm{R}} Z_{\mathrm{R}}(\varphi \approx 90 \mathrm{deg})$, i.e., when the scan angle is small, as in space applications, both conditions can be practically fulfilled by the two orthogonal polarizations of a dual-polarized feed. Thus, multiple beams in dual polarization can be generated. However, when $\varphi$ angle of the beam is far from 0 or 90 degrees, as in the lateral beams of the present application, the $\boldsymbol{E}_{f}$ vectors that fulfill the (22) and (23) respectively are not orthogonal. As a result, the two equations cannot be fulfilled at the same time by a dual-polarized feed. In the present case, the central sector can be covered in dual-linear polarization, but the lateral beams cannot be generated in dual-polarization by single feeds. For this reason, alternate polarization is proposed for adjacent beams, in order to introduce polarization isolation.

Finally, the selected feed-horn is $20 \mathrm{dBi}$ gain and its placement causes a near-field illumination of the reflectarray. In fact, the reflectarray elements are in the Fresnel region of the feedhorns, and the far-field model may not be suitable for the analysis and design of the reflectarray. In these cases, the real incident field on the reflectarray surface can be taken into account for a more accurate analysis and design of the antenna, but the model has been checked and is accurate enough in this case [21].

\section{B. Synthesis of the Radiation Pattern}

Once the 3D masks of requirements and the antenna geometry are defined, the phase of the reflection coefficient of each reflectarray element is synthesized in order to fulfill the required pattern for the central beam. The synthesis process is divided into two stages. First, an analytic technique for phase-only synthesis in one dimension [22] is applied. Using this technique, two linear phase distributions are obtained, corresponding to the

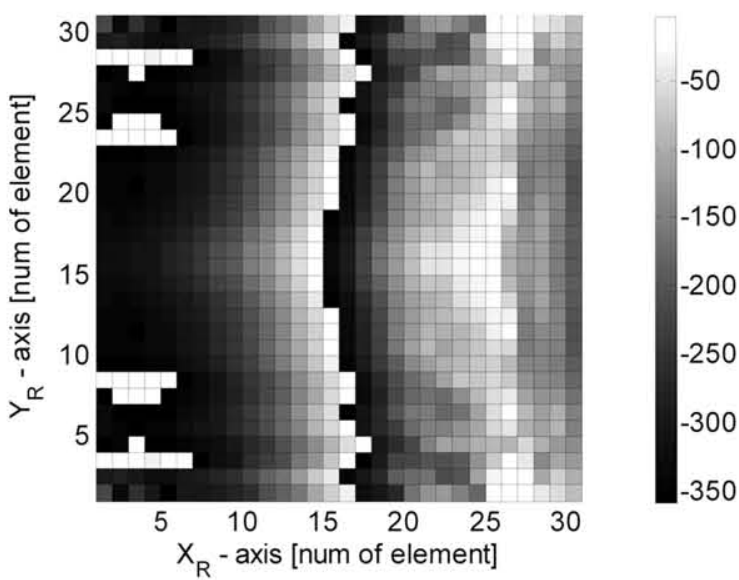

Fig. 3. Synthesized phase distribution of the reflection coefficient for the $X_{\mathrm{R}}$-polarization.

azimuth and elevation requirements respectively. The two-dimensional phase distribution obtained by combining the linear distributions produces a shaped radiation pattern close to the requirements, and is an excellent starting point for a further optimization process.

Secondly, the beam shape is improved by applying an optimization technique based on the Intersection Approach method [23]. A time-efficient implementation of this method adapted to the particular case of reflectarrays and based on Two-dimensional Fast Fourier Transform (2D-FFT), has been applied here [24]. This technique has been used for the design of contouredbeam reflectarrays for space applications with very good results [16]. Here, the technique has been modified and a constraint has been imposed: the phase distribution must be symmetric with respect to the $X_{\mathrm{R}}$-axis, according to the antenna symmetry. If the constrain is not introduced, the radiation patterns of the two lateral beams will not be the same. In fact, one of them can be improved but the other will be worse.

After applying the pattern synthesis technique, an optimized phase distribution of the reflection coefficient is obtained for the central beam at the central frequency. The resulting phase distribution for $X_{\mathrm{R}}$-polarization (V-pol.) is shown in Fig. 3, being the phase distribution for $Y_{\mathrm{R}}$-polarization (H-pol.) very similar. Assuming that the phasing produced on the reflectarray is the same for the three feeds, the phase distribution of Fig. 3 has been used to compute the ideal three-dimensional radiation patterns of the central and one lateral beam, see Fig. 4. The lateral beam shows some distortion because of the small differences in the illumination.

\section{Reflectarray Design and Manufacture}

Once the phase required for the reflection coefficient at each reflectarray element has been determined, the dimensions of the printed patches have to be adjusted to match that phase. In this case, a two-layer configuration, see Fig. 5, has been selected because it provides a good bandwidth for moderate sized reflectarray [5], [25]. Low-loss materials from Arlon have been selected for the reflectarray. CuClad 233LX $\left(\varepsilon_{r}=2.33\right.$; tag $\delta=0.0013$; thickness $=0.787 \mathrm{~mm}$ ) has been chosen as substrate. The layers of printed boards are bonded using a bonding 


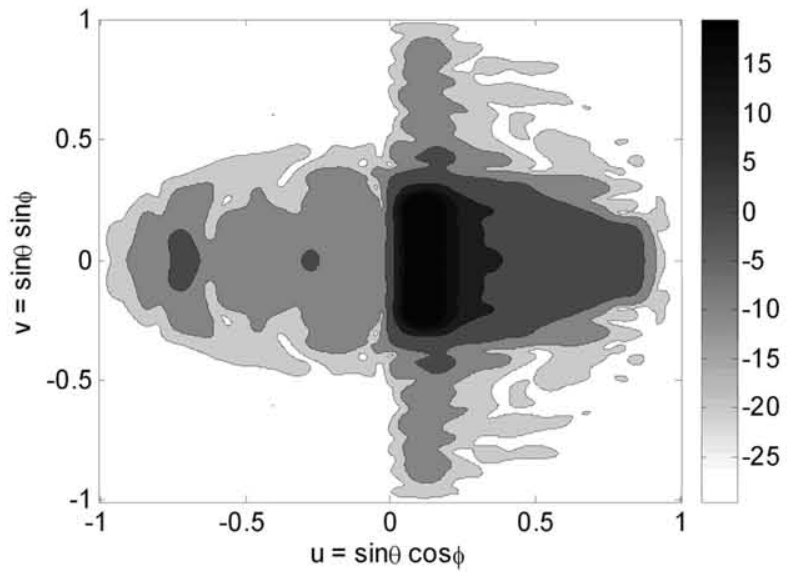

(a)

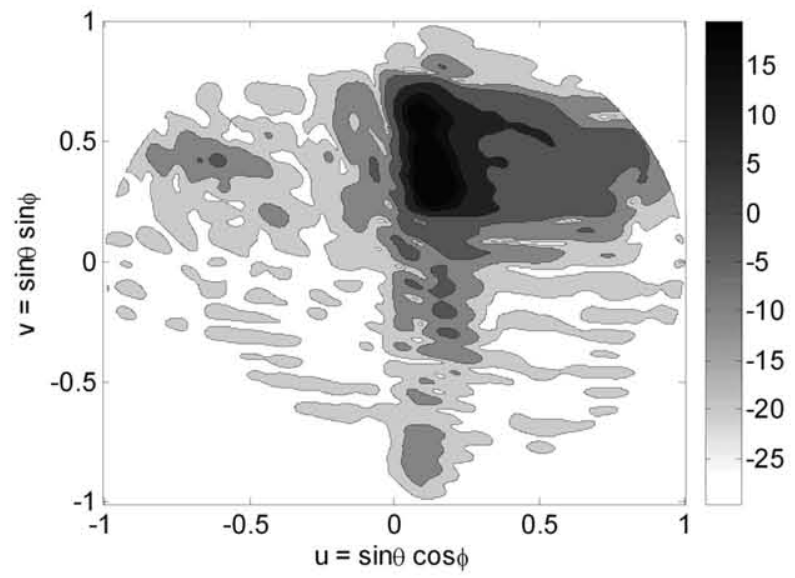

(b)

Fig. 4. Simulated three-dimensional radiation patterns considering ideal phase distribution. The central beam in V-polarization (a) and the lateral beam in H-polarization (b).
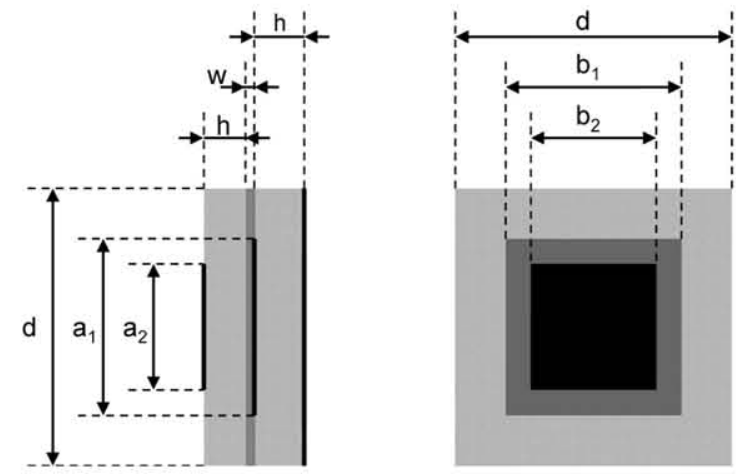

Fig. 5. Periodic cell geometry.

film; CuClad $6250\left(\varepsilon_{r}=2.32\right.$; $\operatorname{tag} \delta=0.0013$; thickness $=0.037 \mathrm{~mm}$ ), which has been taken into account both in the design of the patches and the calculation of the radiation patterns of the reflectarray. The sizes of the two stacked patches fulfill the same ratio, $a_{2}=0.75 a_{1}, b_{2}=0.75 b_{1}$, in every periodic cell.

The behavior of the reflectarray elements have been evaluated, by simulating the periodic cell for different angles of incidence of the impinging wave at the central $(25.5 \mathrm{GHz})$ and extreme frequencies $(24.5 \mathrm{GHz}$ and $26.5 \mathrm{GHz}$ ), see Fig. 6 . The

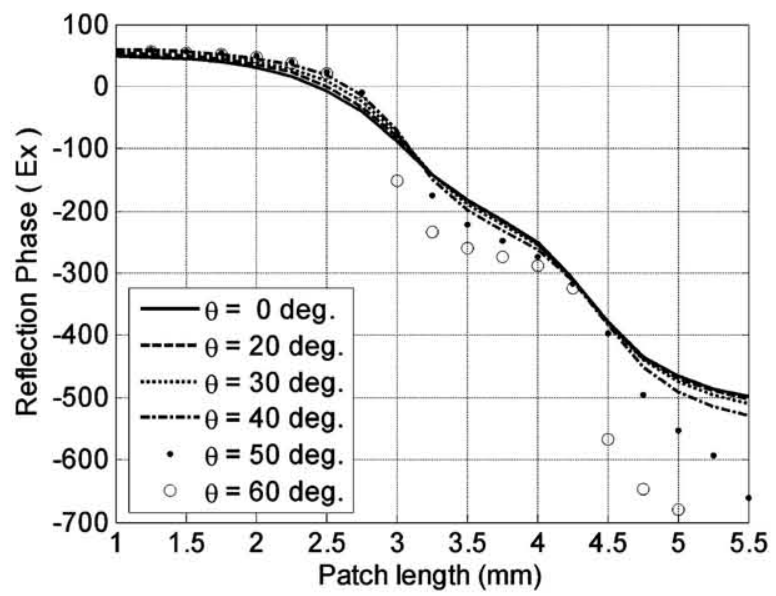

(a)

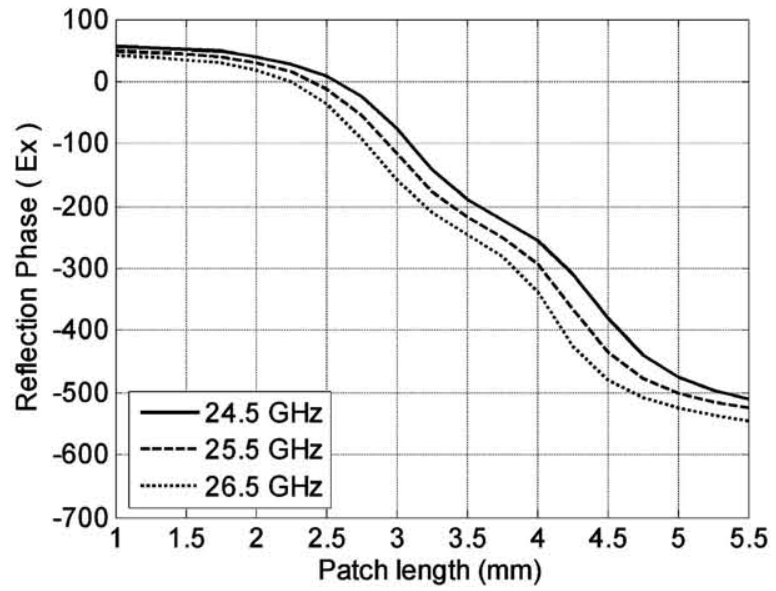

(b)

Fig. 6. Simulated phase of the reflection coefficient of the proposed periodic cell for different angles of incidence at the central frequency (a) and for an incidence angle of 30 degrees at several frequencies (b). The impinging wave is considered on the $X_{\mathrm{R}} Z_{\mathrm{R}}$ plane with $X_{\mathrm{R}}$-polarization (TM-wave).

results shown in Fig. 6 have been obtained considering an incident wave on the $X_{\mathrm{R}} Z_{\mathrm{R}}$ plane $(\varphi=0)$ with $X_{\mathrm{R}}$-polarization according with Fig. 1, which corresponds to a TM-wave. Note that the reflection coefficient of a periodic array depends on the polarization of the impinging wave. Since the phase of the reflection coefficient does not vary too much for incidence angles under 40 degrees, Fig. 6(a), the defined periodic cell is suitable for the present design. Results for a $Y_{\mathrm{R}}$-polarized incident wave have shown a very similar behavior.

The design is carried out at the central frequency and for the central beam using the technique described in [5]. The dimensions of the patches in each cell are adjusted by using an iterative routine that calls the analysis routine until the phase of the reflection coefficient matches the required phase-shift obtained by the synthesis process, see Fig. 3. Each cell is considered to be in a periodic environment and the angle of incidence of the impinging wave at each element is taken into account. Also, the adjustment is carried out for the two dimensions so that the reflectarray is designed in dual linear polarization. The photo-etching mask obtained in this process for the lower layer of the reflectarray is shown in Fig. 7, being the patches of the upper layer scaled by a factor of 0.75 . 


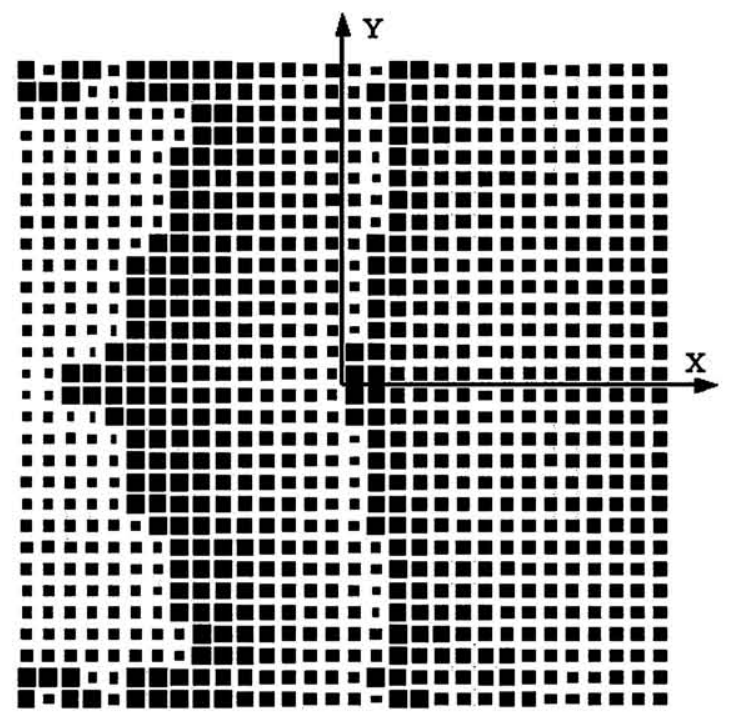

Fig. 7. Photo-etching mask for the lower layer.

A breadboard of the designed antenna has been manufactured. The two layers of printed arrays have been produced by a chemical etching process and the dimensions of the patches have been verified with a profile projector. The layers have been glued by means of a bonding film layer in a curing cycle, according to the manufacturer specifications. Then, the reflectarray is assembled with a support structure, which ensures the position of the feed-horn and the reflectarray panel. The structure has been designed to allow several positions of the arm and rotations of the feed horn, in order to measure the central and lateral beams in both polarizations.

\section{RESULTS}

The design technique has been validated by comparing the different radiation pattern obtained from simulations and measurements. Since the antenna has been designed for three beams in dual polarization, the central and lateral beams have been simulated and measured in vertical and horizontal polarizations (Vand $\mathrm{H}-)$. The reflectarray has been analyzed through the technique described in Section II, considering local periodicity and the angle of incidence on each element. The antenna has also been measured in an anechoic chamber using a compact range system, see Fig. 8. Main cuts of the beams, elevation and azimuth, have been obtained in $\mathrm{V}$ - and $\mathrm{H}$ - polarizations in order to evaluate all the cases.

The antenna can work in two different configurations using alternate polarization for adjacent beams: V-H-V and $\mathrm{H}-\mathrm{V}-\mathrm{H}$. In the first case, the central beam works in the horizontal polarization while the lateral beams are in the vertical polarization, being in the second case the other way around. For the measurement of both polarizations, the feed-horn is rotated. Note that sectors of 180, 270 and 360 degrees in azimuth can be covered by combining the two possible configurations to keep polarization alternation.

The first configuration that has been considered is the V-H-V. So, the central and lateral beams work in horizontal (H-pol.) and vertical (V-pol.) polarizations respectively. The main cuts of the radiation pattern obtained by measurements and simulations are

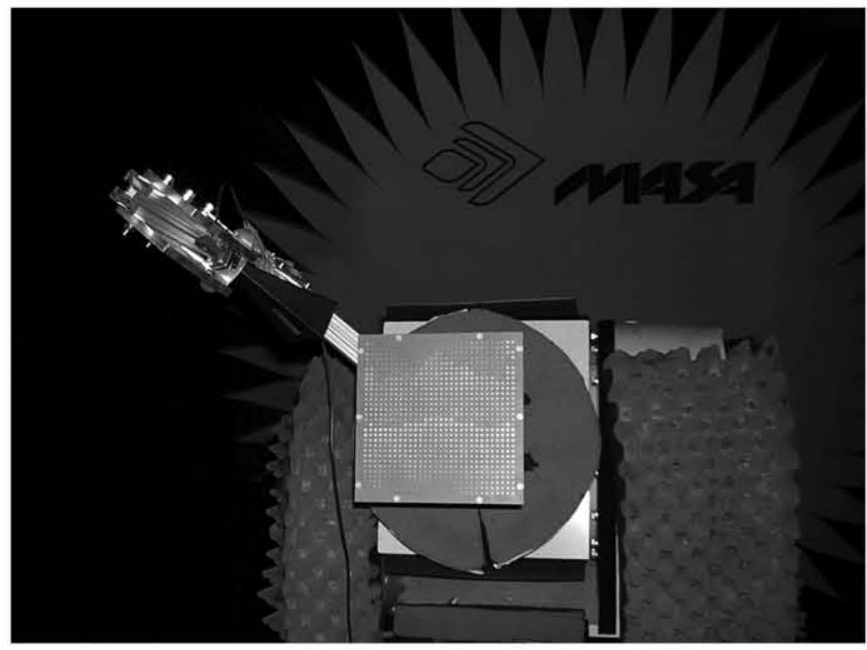

Fig. 8. Breadboard in the anechoic chamber in V-pol. and lateral beam configuration.

compared at Fig. 9 at central frequency, showing a fairly good agreement for both lateral and central beams, particularly in the coverage regions. The radiation patterns are computed and measured in gain $(\mathrm{dBi})$ including ohmic losses. The measured patterns reproduce well the beam shaping and fulfill the minimum gain requirements in more than $90 \%$ of the coverage in all the cases, showing a low level of side lobes.

These results show that the analysis technique provides fairly good predictions for the copolar radiation patterns in the coverage regions and provides reasonable estimations of crosspolar level. However, there are some disagreements, as a decrease of gain in the elevation radiation pattern for $\theta=18 \mathrm{deg}$, Fig. 9(c), some ripple in the coverage regions and also some discrepancies in the side-lobe region. These slight deviations can be caused by several factors: first, the use of a far-field ideal model for the feed-horns instead of the actual incident field; second, the tolerances in the manufacturing process; and third, the local periodicity approach used in the analysis of the reflectarray.

Because of the illumination in the Fresnel zone of the feeds, a source of distortion comes from the use of an ideal far-field model for the feeds. The incident field obtained by the far-field model was compared with the real near field of the horn obtained from measurements for the central feed [21], showing slight differences. The differences between both models for the incident field are more significant for the lateral beams, because of the larger angles of incidence. This fact justifies a better agreement for the central beam than for the lateral ones. Therefore some improvement can be achieved in the theoretical predictions by the use of the actual incident field on each reflectarray element.

Concerning the effects of the tolerance errors on the radiation patterns, it has been demonstrated in previous breadboards that the most critical parameters are the thickness and dielectric constant of the dielectric layers, and patch dimensions. In the results presented in [8] a 55-micron in excess average error of the patch dimensions produced a frequency shift from 25.5 $\mathrm{GHz}$ to $25 \mathrm{GHz}$ and some distortion in the radiation patterns. In the present breadboard, the patch dimensions have been measured in some samples showing an average error of 14 microns 


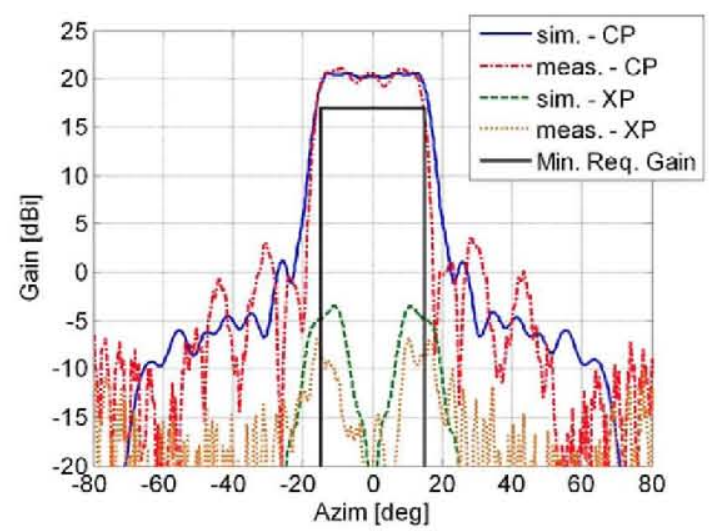

(a)

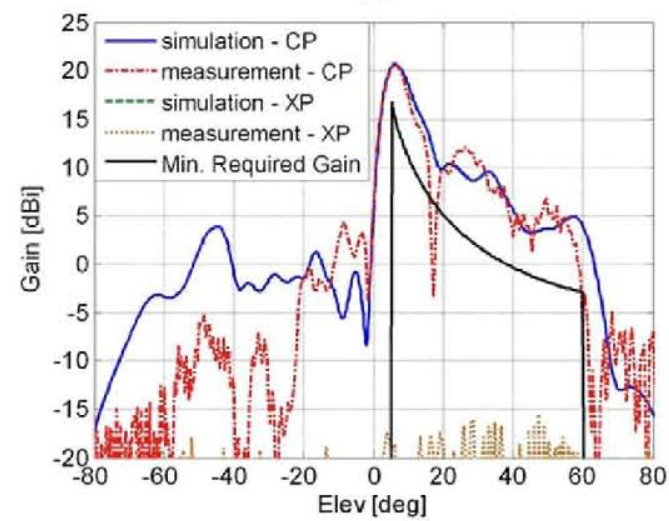

(c)

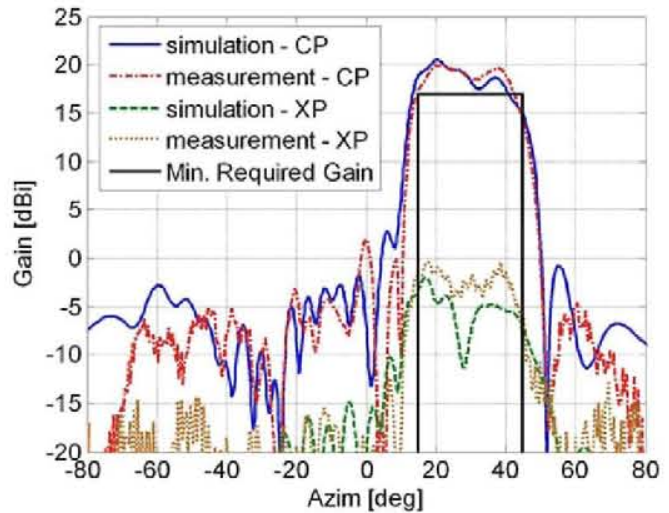

(b)

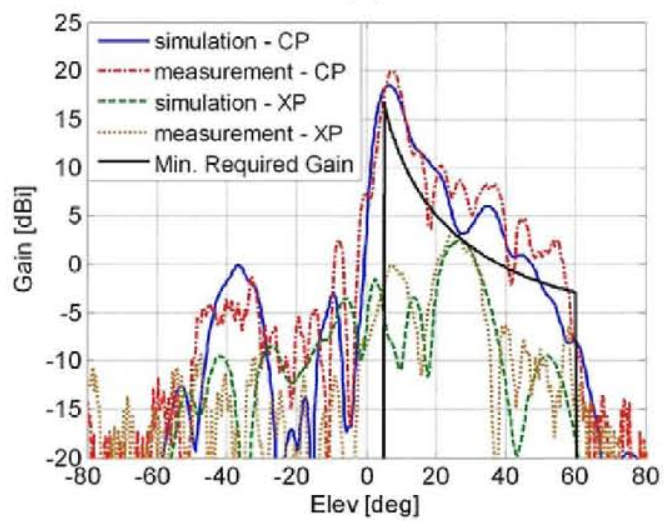

(d)

Fig. 9. Simulations (copolar and crosspolar in solid and dashed lines, respectively) and measurements (copolar and crosspolar in dash-dot and dotted lines, respectively) at the central frequency (25.5 GHz). Central beam in H-polarization (a) and (c) and lateral beam in V-polarization (b) and (d). Main cuts in azimuth (a) and (b) and elevation (c) and (d). Minimum required gain in solid line.

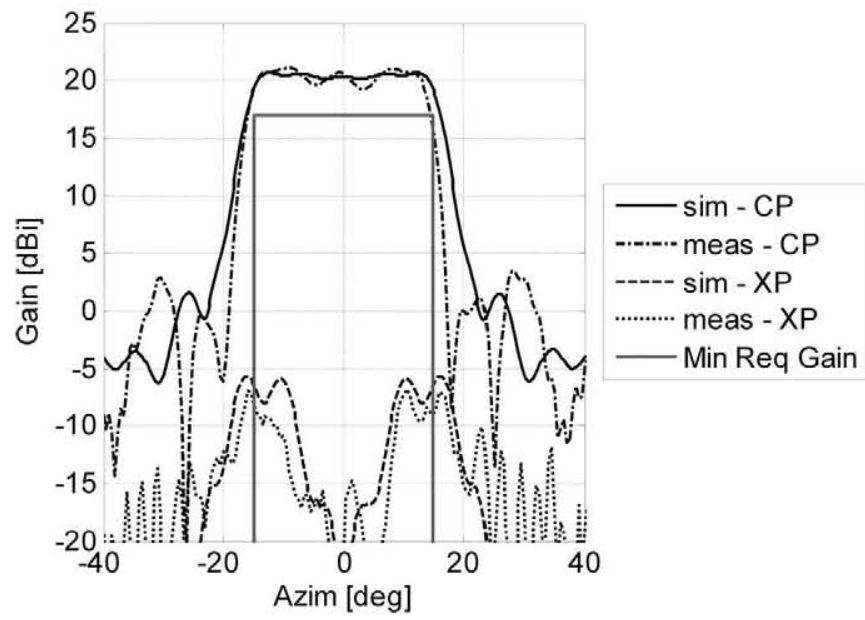

Fig. 10. Simulations taken into account the error in substrate thickness (copolar and crosspolar in solid and dashed lines respectively) and measurements (copolar and crosspolar in dash-dotted and dotted lines respectively) at the central frequency $(25.5 \mathrm{GHz})$. Main azimuth cut of central beam in $\mathrm{H}$-polarization. Minimum required gain in light-colored solid line.

(maximum is 30 microns) below the nominal values. These errors are within a small tolerance range and have not produced a deviation of the frequency band, but they may be responsible for small deviation on the radiation patterns, since the variation of the phase with the dimension is not linear. The thickness of the Cuclad has been measured as $0.81 \mathrm{~mm}$, which is 40 microns

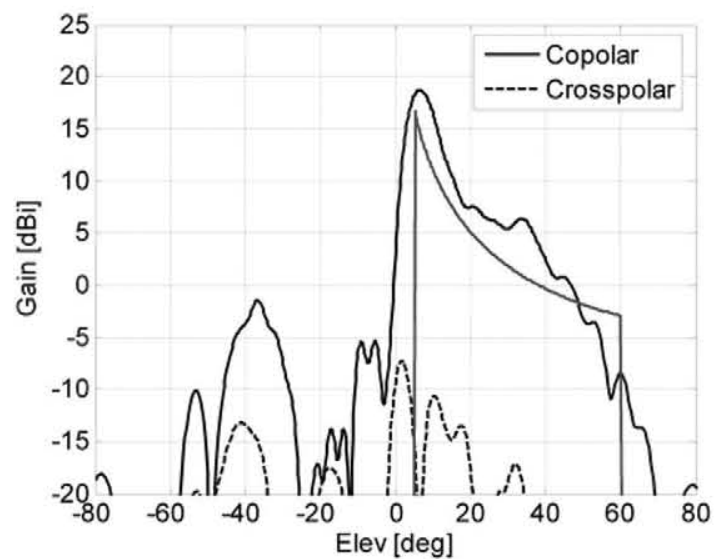

Fig. 11. Simulated radiation pattern of the lateral beam in V-pol. considering ideal phase shifters and a rotation of 5 degrees of the feed horn.

thicker than the nominal value. This error has been taken into account in further simulations and better agreement is found between these results and measurements, mainly in crosspolar pattern, see Fig. 10. These uncertainties in the design process can be overcome by measuring the losses and phase-shift of two reflectarray elements in a waveguide simulator. These results have provided accurate agreement between simulations and measurements, taking into account the waveguide environment, see page 54 in [1]. 


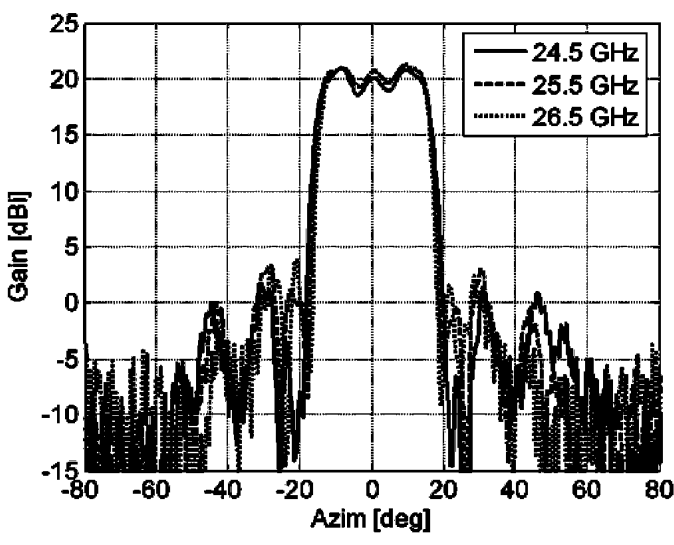

(a)

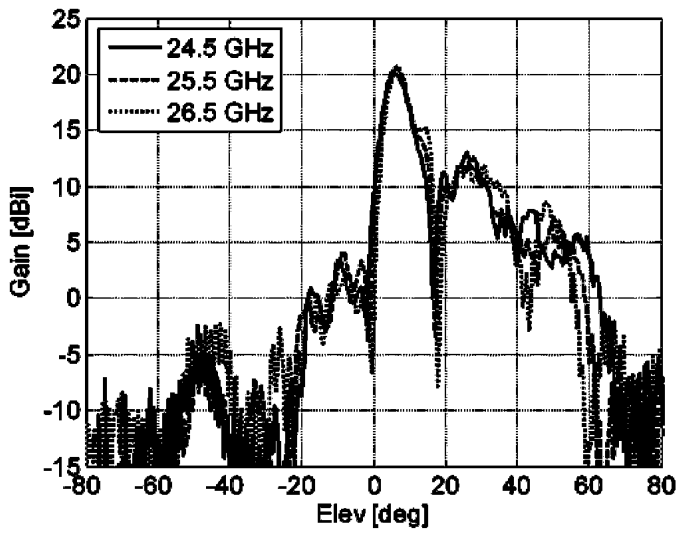

(c)

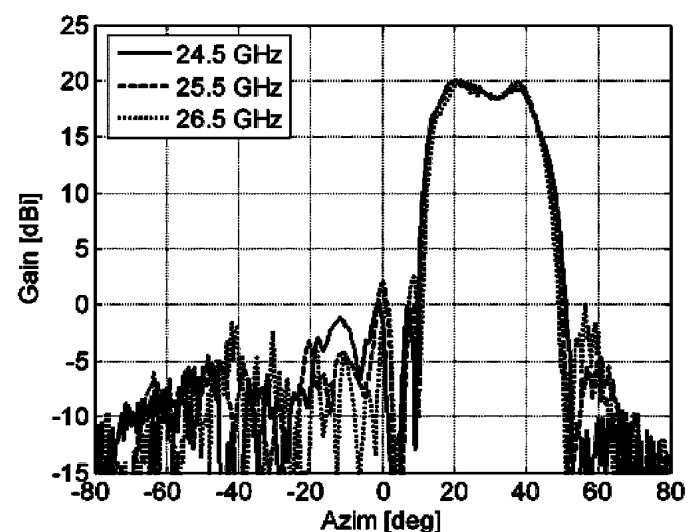

(b)

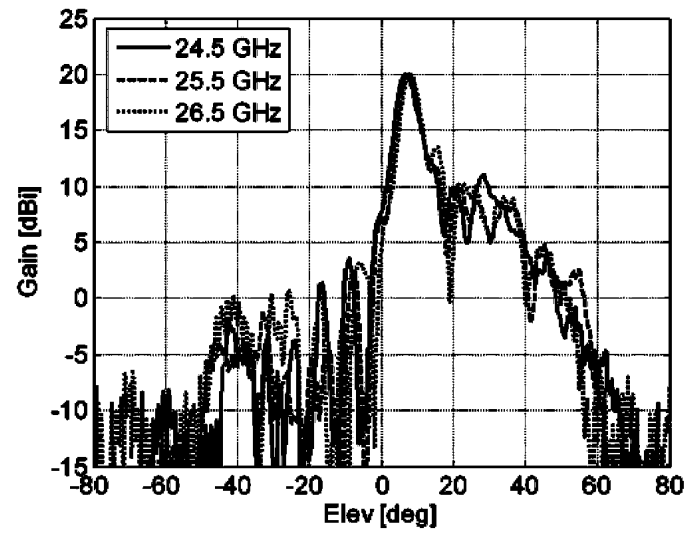

(d)

Fig. 12. Measurements at three frequencies (24.5, 25.5, and 26.5 GHz) of the central beam in H-pol. (a) and (c) and lateral beam in V-pol. (b) and (d). Main cuts in azimuth (a) and (b) and elevation (c) and (d).

It has been demonstrated in a previous breadboard in $\mathrm{Ku}-\mathrm{bad}$ that the local periodicity approach gives very good predictions for contoured beam reflectarrays [16]. In this approach, all the mutual couplings are taken into account in the analysis, assuming that all the neighbors are identical. The approach is accurate when the variation of the patch dimensions is smooth from one element to the next, but it is not appropriate in the zones where there are 360-degree steps, and on the edges of the reflectarray. However, the elements surrounded by patches of very dissimilar dimensions only represent a small percentage of the total surface, see the mask in Fig. 7, so the effect of their inaccuracies is small. Also, the amplitude of the incident field is very low in the elements near the reflectarray edge, and therefore their contribution to the radiation patterns is practically negligible. In conclusion, the small discrepancies observed between measured and simulated radiation patterns should be produced by a combination of the three factors considered above.

As shown in Fig. 9(d) the crosspolar pattern of the lateral beam is too high at an elevation angle of 23 degrees, which corresponds to $\theta=37$ degrees and $\phi=51$ degrees. This is the direction of specular radiation of the lateral feed supposing the reflector as a metal plane, see Table II. The crosspolar radiation is produced by two different facts, one is the antenna geometry, and the other the reflectarray elements, as explained in Section II. The component caused by the geometry can be evaluated and reduced with a rotation of the feed horn around the $Z_{\mathrm{F}}$ axis which does not modify the copolar pattern, see Fig. 11 .
This simulation is carried out with a 5-degree rotation of the feed around the $Z_{\mathrm{F}}$ axis and assuming that the elements of the reflectarray are ideal phase shifters so that the elements $\rho_{x y}$ and $\rho_{y x}$ of the matrix $\boldsymbol{R}$ in (4) are zero. However, the crosspolar radiation generated by the patches is more important and it cannot be compensated with the rotation of the feed, being necessary the application of other techniques, as explained in [26].

Finally, the antenna has been measured at the central (25.5 GHz) and extreme (24.5 GHz and $26.5 \mathrm{GHz}$ ) frequencies of the band. Both central and lateral beams have been measured in this configuration, showing good behavior in band, see Fig. 12. The beam shaping is maintained in the entire frequency band, as well as the gain and secondary lobes.

The antenna has been also evaluated in the dual configuration, H-V-H (central and lateral beams in vertical and horizontal polarizations respectively). The results obtained in this configuration are very similar to those showed for the previous configuration.

\section{CONCLUSION}

A design procedure for reflectarrays with multiple feeds and multiple shaped-beams has been presented. For the validation of the method, a two-layer printed reflectarray with patches of varying size has been designed, manufactured and measured. The reflectarray is proposed as an antenna for LMDS central station application with three feed horns and three independent shaped beams. Each beam can work in any linear polarization 
so that there are two possibilities of alternate polarization for the beams, which permit the channel capacity to be increased. The antenna has been manufactured and tested in both linear polarizations, and the results show a good agreement with the shaping requirements. These results demonstrate the capability of reflectarrays to generate simultaneous shaped beams in the one feed per beam basis.

\section{REFERENCES}

[1] J. Huang and J. A. Encinar, Reflectarray Antennas. Piscataway, NJ/New York: IEEE Press/Wiley, 2007.

[2] J. A. Encinar, E. Carrasco, and M. Arrebola, , A. Tazón, Ed., "Analysis, Design and Applications of Multi-Layer Printed Reflectarrays," in Advances on Antennas, Reflectors and Beam Control. Kerala, India: Research Signpost, 2005, ch. 2, pp. 25-51.

[3] D. C. Chang and M. C. Huang, "Feasibility study of erecting cosecant pattern by planar microstrip reflectarray antenna," in Proc. AMPC'93, 1993, vol. 2, pp. 19.20-24.

[4] D. M. Pozar, S. D. Targonski, and R. Pokuls, "A shaped-beam microstrip patch reflectarray," IEEE Trans. Antennas Propag., vol. 47, no. 7, pp. 1167-1173, Jul. 1999.

[5] J. A. Encinar, "Design of two-layer printed reflectarrays using patches of variable size," IEEE Trans. Antennas Propag., vol. 49, no. 10, pp. 1403-1410, Oct. 2001.

[6] J. A. Encinar and J. A. Zornoza, "Three-Layer printed reflectarrays for contoured beam space applications," IEEE Trans. Antennas Propag., vol. 52, pp. 1138-1148, May 2004.

[7] R. Leberer and W. Menzel, "A dual planar reflectarray with synthesized phase and amplitude distribution," IEEE Trans. Antennas Propag., vol. 53, pp. 3534-3539, Nov. 2005.

[8] J. A. Zornoza, R. Leberer, J. A. Encinar, and W. Menzel, "Folded multi-layer microstrip reflectarray with shaped pattern," IEEE Trans. Antennas Propag., vol. 54, pp. 510-518, Feb. 2006.

[9] L. Schulwitz and A. Mortazawi, "A compact dual-polarized multibeam phased-array architecture for millimeter-wave radar," IEEE Trans. Microw. Theory Tech., vol. 53, no. 11, pp. 3588-3594, Nov. 2005.

[10] A. Jacomb-Hood and E. Lier, "Multibeam active phased arrays for communications satellites," IEEE Microw. Mag., vol. 1, no. 4, pp. 40-47, Dec. 2000.

[11] A. K. Bhattacharyya, Phased Array Antennas-Floquet Analysis, Synthesis, BFNs, and Active Array Systems. New York: Wiley, 2006.

[12] P. Balling, K. Tjonneland, L. Yi, and A. Lindley, "Multiple contoured beam reflector antenna systems," presented at the Proc. IEEE Antennas Propagation Soc. Int. Symp., Ann Arbor, MI, Jun. 28-Jul. 21993.

[13] W. Menzel, M. Al-Tikriti, and R. Leberer, "A 76 GHz multiple-beam planar reflector antenna," in Proc. 32nd Eur. Microwave Conf., Milano, Italy, Oct. 2002, vol. III, pp. 977-980.

[14] J. A. Encinar and J. A. Zornoza, "Multi-beam reflectarrays," presented at the Progress in Electromagnetics Research Symp.-PIERS, Boston, MA, Jul. 21-25, 2002.

[15] M. Barba, J. E. Page, and J. A. Encinar, "Multi-beam printed arrays and reflectarrays," in COST-284 Regular Meeting, Dubrovnik, Croatia, Oct. 11, 2005.

[16] J. A. Encinar, L. Datashvili, J. A. Zornoza, M. Arrebola, M. Sierra-Castañer, J. L. Besada, H. Baier, and H. Legay, "Dual-polarization dualcoverage reflectarray for space applications," IEEE Trans. Antennas Propag., vol. 54, pp. 2827-2837, Oct. 2006.

[17] M. Thiel and W. Menzel, "A multiple-beam sector antenna with a dual planar reflectarray arrangement," in Eur. Radar Conf. EuRAD2006, Manchester, U.K., Sep. 2006, pp. 53-56.

[18] European Standard (Telecommunications Series), Fixed radio Systems; Point-to-Multipoint Antennas; Antennas for Point-to-Multipoint Fixed Radio Systems in the $11 \mathrm{GHz}$ to $60 \mathrm{GHz}$ Band; Part 2: $24 \mathrm{GHz}$ to $30 \mathrm{GHz}$, ETSI EN 301215 v1.3.1, Jun. 2002.

[19] C. Wan and J. A. Encinar, "Efficient computation of generalized scattering matrix for analyzing multilayered periodic structures," IEEE Trans. Antennas Propag., vol. 43, pp. 1233-1242, Nov. 1995.

[20] A. Ludwig, "The definition of cross polarization," IEEE Trans. Antennas Propag., vol. 21, pp. 1116-119, Jan. 1973.

[21] M. Arrebola, J. A. Encinar, Y. Álvarez, F. Las-Heras, and W. Menzel, "Design of a reflectarray with three shaped beams using the near-field radiated by the feeds," in First Eur. Conf. Antennas Propag.-EuCAP, Nice, France, Nov. 6-10, 2006.

[22] A. Chakraborty, B. N. Das, and G. S. Sanyal, "Beam shaping using nonlinear phase distribution in a uniformly spaced array," IEEE Trans. Antennas Propag., vol. 30, pp. 1031-1034, Sep. 1982.
[23] O. Bucci, G. Franceschetti, G. Mazzarella, and G. Panariello, "Intersection approach to array pattern synthesis," Proc. Inst. Elec. Eng., vol. 137, no. 6, pt. H, pp. 349-357, Dec. 1990.

[24] J. A. Zornoza and J. A. Encinar, "Efficient phase-only synthesis of contoured beam patterns for very large reflectarrays," Int. J. RF Microw. Comput.-Aided Eng., pp. 415-423, Sep. 2004.

[25] D. M. Pozar, "Bandwidth of reflectarrays," Electron. Lett., vol. 39, no. 21, pp. $1490-1491$, Oct. 2003.

[26] J. A. Encinar and M. Arrebola, "Reduction of cross-polarization in contoured beam reflectarrays using a three-layer configuration," in Proc. IEEE Antennas Propagation Soc. Int. Symp., Honolulu, HI, Jun. 10-15, 2007.

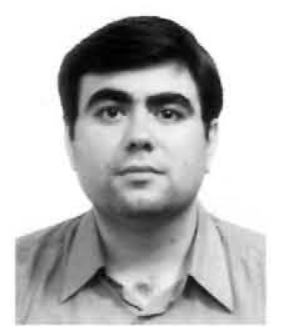

Manuel Arrebola (S'99-M'07) was born in Lucena, Córdoba, Spain. He received the Ingeniero de Telecomunicación degree from Universidad de Málaga (UMA), Málaga, Spain, in 2002. He is currently working toward the Ph.D. degree at the Universidad Politécnica de Madrid (UPM), Madrid, Spain.

From 2003 to 2007, he was with the Electromagnetism and Circuit Theory Department, UPM as a Research Assistant. From August to December 2005, he was with the Microwave Techniques Department at Universität Ulm, Ulm, Germany, as a Visiting Scholar. In December 2007, he joined the Electrical Engineering Department, Universidad de Oviedo, Gijón, Spain, where he is an Assistant Professor. His current research interests include analysis and design techniques of printed reflectarray antennas.

Mr. Arrebola was co-recipient of the 2007 S. A. Schelkunoff Transactions Prize Paper Award given by IEEE Antennas and Propagation Society.

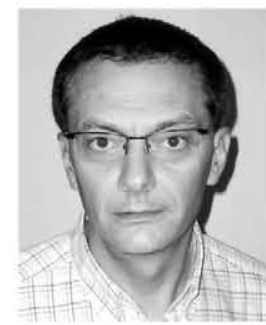

José A. Encinar (S'81-M'86) was born in Madrid, Spain. He received the Electrical Engineer and Ph.D. degrees, both from Universidad Politécnica de Madrid (UPM), in 1979 and 1985, respectively.

Since January 1980, has been with the Applied Electromagnetism and Microwaves Group, UPM, as a Teaching and Research Assistant from 1980 to 1982, as an Assistant Professor from 1983 to 1986, and as Associate Professor from 1986 to 1991. From February to October 1987, he stayed at Polytechnic University, Brooklyn, NY, as a Postdoctoral Fellow of the NATO Science Program. Since 1991, he is a Professor of the Electromagnetism and Circuit Theory Department, UPM. In 1996, he was with the Laboratory of Electromagnetics and Acoustics, Ecole Polytechnique Fédérale de Lausanne (EPFL), Switzerland, as a Visiting Professor. His research interests include numerical techniques for the analysis of multilayer periodic structures, design of frequency selective surfaces, printed arrays and reflectarrays. He has published more than one hundred journal and conference papers, and he is holder of three patents on array and reflectarray antennas.

Prof. Encinar was a co-recipient of the $2005 \mathrm{H}$. A. Wheeler Applications Prize Paper Award and the 2007 S. A. Schelkunoff Transactions Prize Paper Award, given by the IEEE Antennas and Propagation Society.

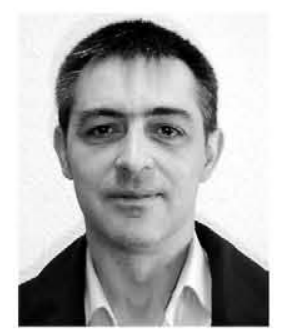

Mariano Barba was born in Murcia, Spain. He received the Ingeniero de Telecomunicación and Ph.D. degrees from the Universidad Politécnica de Madrid, Madrid (UPM), Spain, in 1990 and 1996, respectively.

From 1991 to 1994, he was with the Depto. de Electromagnetismo y Teoría de Circuitos (UPM), as Postgraduate Researcher. During this time he developed numerical methods and algorithms to characterize and design microwave passive devices. From 1994 to 2003, he has been involved in the R\&D departments of several companies and institutions developing antennas and microwave circuits for space and terrestrial communication applications. In 2003, he joined to the Depto. de Electromagnetismo y Teoría de Circuitos, (UPM), as a, Associate Professor. His research interests include the analysis, characterization and design of antenna and microwave circuits. He is currently involved in the development and design of multibeam and reconfigurable antennas. 